\title{
Conséquences de la libéralisation des marchés sur les opérateurs de la filière laitière au Maroc
}

\author{
M.T. Sraïri ${ }^{1 *}$ A. Chohin Kuper ${ }^{2}$
}

\begin{abstract}
Mots-clés
Lait de vache - Libéralisation des échanges - Qualité - Elevage - Maroc.
\end{abstract}

\begin{abstract}
Résumé
La filière laitière marocaine a connu une évolution mouvementée depuis le début des années 1970 jusqu'à nos jours. La situation initiale, très protégée, se caractérisait par un interventionnisme étatique prononcé car il fallait pallier dans l'urgence la rareté du lait face à une demande en plein essor. A partir de 1980, I'application de mesures d'ajustement structurel a progressivement supprimé toute forme de subvention. De tels bouleversements et la rapidité avec laquelle ils se sont enchaînés ont eu des répercussions marquées sur l'organisation de la filière, en particulier dans les élevages de bovins laitiers où les caractéristiques de la production ont notablement évolué. Actuellement, un pas de plus va être franchi avec la préparation à l'entrée en vigueur des accords de libre-échange avec l'Union européenne. Ceci va induire la fin de toute protection et une concurrence accrue entre produits laitiers locaux et importés sur le marché marocain. Dans cette optique de libéralisation et compte tenu du renchérissement actuel des prix des intrants agricoles sur les marchés internationaux, une nécessaire mise à niveau des outils de production et de régulation au sein de la filière est attendue avec des conséquences certaines pour l'ensemble de ses opérateurs. Le maintien des activités de production, de collecte et de transformation du lait au Maroc, dans des conditions propices à leur développement durable, est en jeu : recherche de productivité optimale et de produits de qualité, efficience de la valorisation de l'eau, distribution équitable des revenus de la filière à tous ses intervenants, avec pour objectif un prix au consommateur en rapport avec le pouvoir d'achat.
\end{abstract}

\section{INTRODUCTION}

L'approvisionnement alimentaire des populations des pays du Sud, caractérisées par une croissance démographique rapide et l'évolution des modes de consommation liée à un rythme d'urbanisation soutenu, fait peser de très lourdes responsabilités sur les pouvoirs publics. Celles-ci sont plus particulièrement ressenties pour les produits animaux, comme le lait, pour lequel les perspectives d'augmentation de la demande mondiale devraient générer de vives tensions (10). Si les intrants nécessaires à l'essor de la

1. Département des productions et biotechnologies animales, Institut agronomique et vétérinaire Hassan II, BP 6202, Madinate Al Irfane, 10 101, Rabat, Maroc.

2. CAP Rural, Meknès, Maroc.

* Auteur pour la correspondance

Tél. : + 21237771745 ; fax : + 21237778135

E-mail : mt.srairi@iav.ac.ma production de lait sont déjà source d'inquiétude, notamment les tourteaux de protéagineux et les céréales (4), la possibilité de recourir à des importations de lait est, elle aussi, devenue problématique : les cours mondiaux ont explosé en raison d'une demande sans cesse croissante et qui risque de durer (18).

Simultanément, la libéralisation du commerce génère dans les pays du Sud l'obligation d'ouverture imminente des marchés et la renonciation, à très court terme, à la protection des filières agricoles locales. La libéralisation comprend aussi les fluctuations des cours mondiaux (des matières premières, denrées alimentaires ou intrants) dues à l'évolution de la demande ou encore aux aléas climatiques. Le Maroc s'est ainsi engagé depuis plus d'une vingtaine d'années dans l'adaptation de son agriculture au processus de la mondialisation. Après la phase euphorique des années 1970, caractérisée par l'intervention de l'Etat dans le cadre de politiques productivistes et protectionnistes (7), un changement de cap est 
intervenu avec l'application d'un programme d'ajustement structurel (PAS) au début des années 1980, sous l'injonction des bailleurs de fonds internationaux (29). Plus récemment, le pays est entré dans des négociations d'accords de libre-échange (ALE) avec l'Union européenne, après celui conclu en 2004 avec les Etats-Unis d'Amérique. Pour les différents opérateurs de la filière bovine laitière, ces rapides évolutions ont déjà eu des répercussions marquées. Elles vont aussi induire des changements profonds de mentalités et de pratiques, imposant au passage la mise à niveau par rapport à des standards internationaux de performances et de qualité. Cet article fait le point sur les changements escomptés au niveau de la filière laitière marocaine par la libéralisation des marchés, après avoir dressé le bilan des mutations depuis l'Indépendance du pays, en 1956 (de l'approche dirigiste et volontariste qui atteint son apogée avec le «plan laitier » de 1975, au désengagement des services techniques suite à l'application des mesures du PAS). Les priorités et options pour faire face aux évolutions en cours (libéralisation de la filière lait et accroissement des prix des intrants) et instaurer une filière durable, apte à approvisionner le marché efficacement tout en rémunérant équitablement ses opérateurs, seront discutées à la fin de cet article.

\section{METHODOLOGIE}

En vue de présenter l'éventail des évolutions des outils de la politique laitière au Maroc, un ensemble de documents officiels ont été consultés : le «plan laitier » de 1975 (15), les évaluations annuelles des réalisations de la filière et la discussion des perspectives du secteur depuis le désengagement de l'Etat qui devraient servir de plan directeur pour la période s'étalant de 2000 à 2020 (14). Des diagnostics plus ponctuels de situations d'élevages de bovins, de leurs performances et de la qualité de leurs productions $(22,23$, 24) et des réalisations de la collecte laitière et des industries de la transformation ont aussi été utilisés (6). Les évolutions de la consommation du lait et de ses dérivés ont été analysées à partir des résultats des enquêtes de consommation des ménages (11). Les perspectives de la filière laitière, issues des changements récents de cap de la politique agricole au Maroc et de l'engagement des négociations de libre-échange avec l'Union européenne, sont le fruit de discussions avec les différents opérateurs du secteur, ainsi que de tendances plus globales identifiées à l'échelle de toute la région maghrébine (21). L'approche filière fait référence à la méthodologie développée par les organismes comme le Cirad et la FAO, et l'Union européenne $(8,17)$, où les aspects techniques, économiques et institutionnels sont pris en compte. Dans cet article, l'analyse de la filière laitière marocaine et de ses évolutions sera réalisée pour chacun des maillons, pris d'abord individuellement. Par la suite, l'articulation entre les différents opérateurs de la filière sera considérée. Les priorités et options sont finalement discutées dans une perspective plus globale.

\section{- EVOLUTIONS RECENTES DES DIFFERENTS OPERATEURS DE LA FILIERE LAITIERE}

La filière laitière au Maroc se décompose en quatre maillons distincts groupant divers opérateurs. De l'aval à l'amont de la filière, les intervenants se présentent comme suit : les consommateurs approvisionnés par des unités de distribution (épiceries, grandes surfaces, supérettes...) ; les unités de transformation du lait cru (unités industrielles et coopératives) ; les organismes de collecte du lait qui assurent le lien entre des exploitations dispersées et les industriels ; et une diversité de fermes d'élevage bovin, dont la contribution à la production de lait est très variable selon la technicité mise en œuvre, les races exploitées, les ressources alimentaires mobilisées. Dans la présentation du maillon de la collecte du lait, la sous-filière informelle du colportage sera aussi évoquée (figure 1).

Pour situer l'état actuel des différents acteurs de la filière laitière marocaine et leurs marges futures d'opération, un bref rappel de leurs évolutions depuis l'Indépendance est au préalable nécessaire (encadré 1), avec un bilan des mesures constituant le « plan laitier ». Schématiquement, il est possible de retenir que ce plan a permis :

- une hausse palpable et quasi continue (en dehors des épisodes de sécheresse prononcée de 1981 et de 1994) de la production annuelle du lait de vache, avec un taux d'accroissement annuel d'environ 4 p. 100 entre 1975 et 2006, passant de près de 400 à plus de 1300 millions de litres (figure 2), dont plus de 60 p. 100 proviennent des régions irriguées, qui ne représentent pourtant que 15 p. 100 de la superficie agricole utile du pays (figure 3);

- une chute parallèle de la part du cheptel de races locales qui régresse de 90 à 53 p. 100 des effectifs totaux de 1975 à 2005 ,

Encadré 1

HISTORIQUE DE LA FILIÈRE LAITIÈRE DE L'INDÉPENDANCE JUSQU'AU DÉBUT DES ANNÉES 1980

A I'Indépendance, en 1956, les politiques d'élevage bovin laitier au Maroc sont totalement conduites sous la houlette de l'Etat. Les pouvoirs publics héritent d'une conjoncture caractérisée par la quasi-absence $d^{\prime}$ 'une filière laitière organisée, les colons ayant très peu investi dans ce secteur. La situation est alors dominée par un outil de production atomisé, une offre très saisonnière (au printemps, avec l'abondance fourragère des années pluvieuses) et des circuits de valorisation très courts, tandis que plus de $90 \mathrm{p}$. 100 du lait échappent à la transformation industrielle, les villes étant alors approvisionnées par des réseaux d'étables de proximité (Tahiri, 1963).

Face à l'urgence de satisfaire une demande intérieure en croissance, la mise en place d'une politique laitière se concrétise sous l'impulsion d'agronomes et de vétérinaires visionnaires. Elle vise au départ surtout les zones agroécologiques les plus propices, notamment les plaines irriguées du Nord (Projet Sebou, 1961). Peu après, le mouvement se consolide avec la promulgation du Code national des investissements agricoles en 1968 et atteint sa maturité avec le « plan laitier » (ministère de l'Agriculture et de la Réforme agraire, 1975). Ce texte est appuyé par un arsenal législatif encourageant la production, la collecte, la transformation et la consommation du lait. Le plan laitier est aussi renforcé par des mesures de protection : taxation des importations de poudre et soutien au prix du lait, à la ferme.

Des mesures d'accompagnement sont aussi prévues pour favoriser l'accès aux intrants pour les éleveurs : subventions sur les semences fourragères, sur les machines à traire, sur les génisses importées de race laitière, gratuité de l'insémination artificielle, de l'encadrement sanitaire contre les zoonoses (tuberculose, brucellose)... L'aménagement de coûteux ouvrages d'irrigation (barrages et réseaux de distribution en aval), fer de lance de toute la politique agricole, s'accompagne alors de la planification de soles fourragères importantes. L'irrigation doit sécuriser la production de fourrages de qualité (luzerne, maïs et bersim) tout le long de I'année et favoriser l'implantation d'étables laitières performantes, affranchies des cycles de sécheresse récurrents. Indirectement, cela a généré une des caractéristiques majeures de la filière laitière marocaine : un décalage géographique entre les bassins de production, surtout situés dans l'arrière-pays, et les zones de consommation représentées par les grands centres urbains de la côte atlantique. Ceci a bien entendu des conséquences marquées sur le traitement logistique de la collecte du lait et de sa redistribution aux consommateurs. 


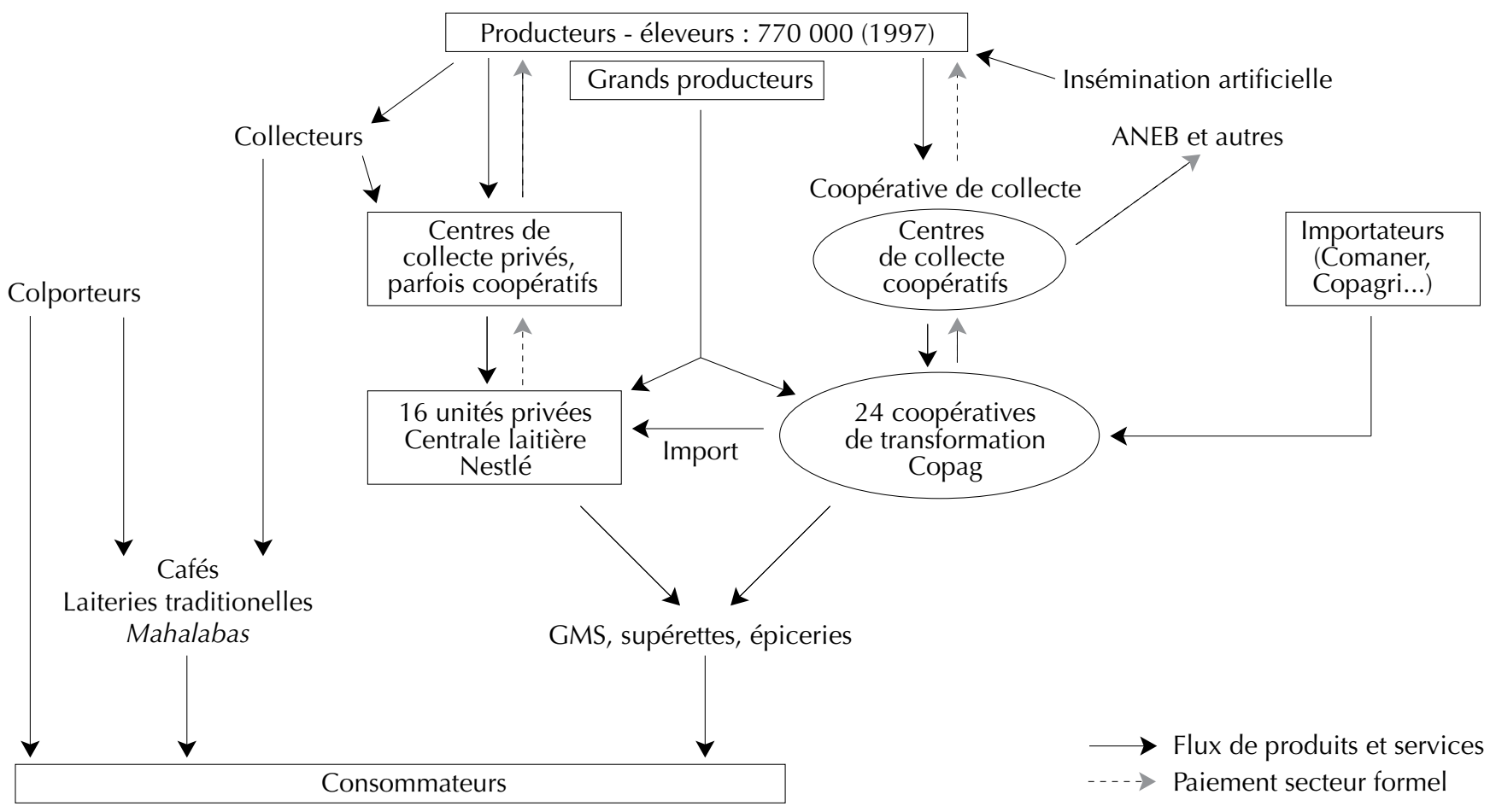

Figure 1 : organisation de la filière laitière au Maroc. Source : auteurs, et ministère de l'Agriculture, du Développement rural et des Pêches maritimes, 2000.

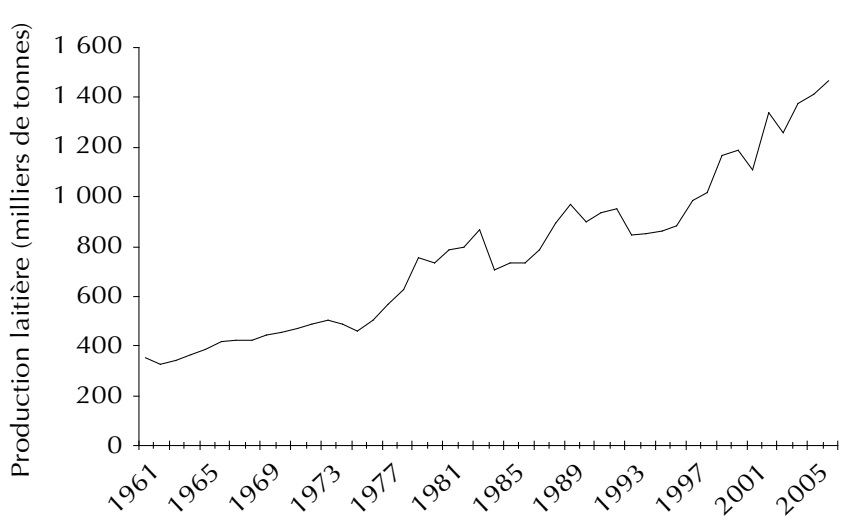

Figure 2 : évolution de la production annuelle de lait de vache au Maroc. D'après Sraïri et coll., 2007.

tandis que le cheptel dit « amélioré » (bovins de race laitière et les produits de leurs croisements avec les races locales) augmente.

Depuis, avec le désengagement de l'Etat, ajustement structurel et libéralisation obligent, les opérateurs se sont pris en main et ont connu des évolutions contrastées qui seront détaillées dans la suite de cet article. Mais à l'évidence, la conséquence directe du désengagement de l'Etat a été la non-réalisation des objectifs initiaux du plan laitier, puisque le niveau de 2000 millions de litres de lait prévu en l'an 2000 est loin d'avoir été atteint en 2007 (production inférieure à 1500 millions de litres).

\section{Consommation des produits laitiers : de fortes disparités}

Malgré l'essor volumétrique de la production laitière durant les trente-cinq dernières années, le niveau de consommation moyen

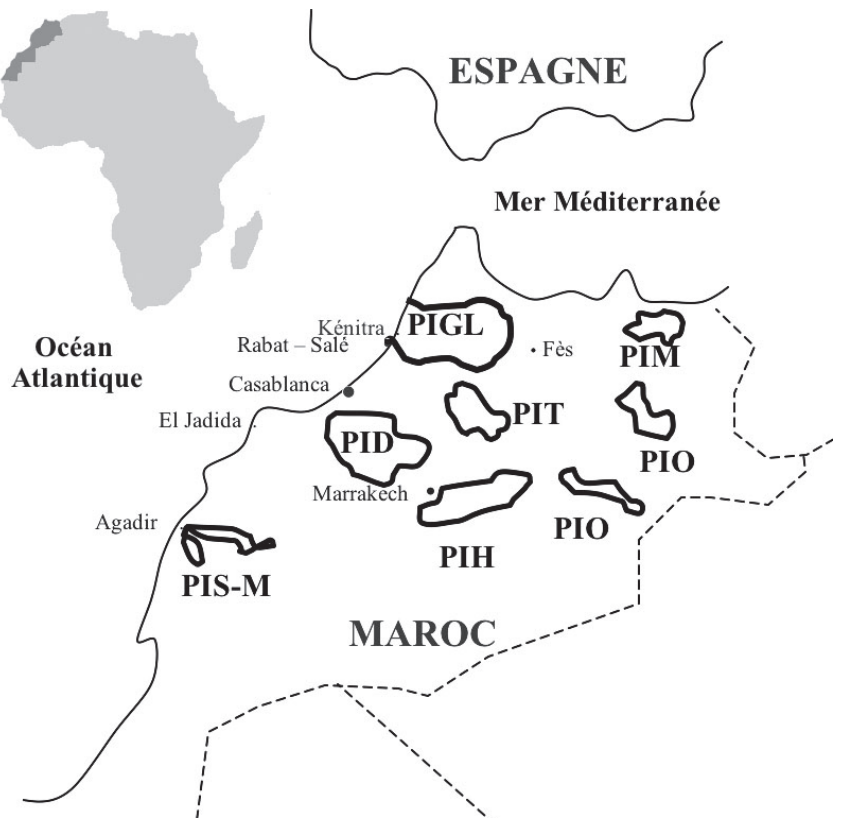

Figure 3 : localisation géographique des grands centres de production et de consommation du lait au Maroc. PID : périmètre irrigué des Doukkala, centre-ouest du pays, 104600 ha irrigués, $15,5 \%$ de la production de lait. PIGL : périmètres irrigués du Gharb et du Loukkos, nord du pays, 132000 ha irrigués, $13,5 \%$ de la production de lait. PIH : périmètre irrigué du Haouz, centre-est du pays, 142000 ha irrigués, $16 \%$ de la production de lait. PIM : périmètre irrigué de la Moulouya, nord-est du pays, 77000 ha irrigués, $3 \%$ de la production de lait. PIO : périmètres irrigués oasiens, est du pays, 65000 ha irrigués, 1,5\% de la production de lait. PIT : périmètre irrigué du Tadla, centre du pays, 109000 ha irrigués, $10,5 \%$ de la production de lait. PIS-M : périmètre irrigué du Souss Massa, sud-ouest du pays, 40000 ha irrigués, $6 \%$ de la production de lait. D'après le ministère de l'Agriculture, du Développement rural et des Pêches maritimes, 2000. 
annuel en lait et dérivés demeure encore limité : 38 équivalents litres de lait/hab. (11). Les augmentations de la production depuis les années 1970 ont à peine accompagné la croissance démographique, impliquant une tendance à la stagnation de la consommation individuelle. A l'échelle du Maghreb, c'est au Maroc que la consommation moyenne de produits laitiers est la plus faible, en comparaison à 95 et 68 équivalents litres de lait/hab./an respectivement en Algérie et en Tunisie. Ces chiffres témoignent aussi de l'ampleur du décalage de la consommation au Maroc par rapport aux recommandations nutritionnelles des organismes internationaux : 90 équivalents litres de lait/hab./an préconisés par la FAO et l'OMS (9). Les différences par rapport aux autres pays maghrébins s'expliquent par une politique privilégiant dès la fin des années 1960 un approvisionnement local plutôt que la reconstitution de lait à partir de poudre importée subventionnée. Elles sont aussi dues à des habitudes alimentaires où le lait n'est pas particulièrement présent dans les préparations culinaires, et surtout aux évolutions récentes du pouvoir d'achat qui font que le lait demeure un produit cher pour la majorité des ménages. Les augmentations du prix du lait pasteurisé de boisson (tableau I) et les hausses encore plus conséquentes des prix des dérivés laitiers ont définitivement associé à ces produits l'image d'une consommation de luxe et ont renforcé les disparités entre les ménages urbains et ruraux. La consommation urbaine atteint en moyenne 53 équivalents litres de lait/hab./an (figure 4), avec des pics de 901 pour les ménages les plus aisés, contre 181 en milieu rural (11). Les variations de la consommation en produits laitiers entre 1985 et 2001 montrent que les ruraux ont même eu tendance à consommer moins de lait (en particulier le petit lait ou lben et le beurre rance ou smen), en raison de ventes plus importantes (1). En outre, les modes de consommation évoluent avec le développement de la restauration hors du domicile familial, notamment dans les laiteries artisanales (qui transforment le lait issu des réseaux de colportage), surtout en milieu urbain, ou encore avec le développement de la distribution moderne (grandes et moyennes surfaces); mais ces changements restent peu étudiés. L'essor des supermarchés avec un élargissement de la gamme des produits offerts, comme en témoigne l'apparition de produits allégés en matières grasses, entraîne aussi une diversification de la consommation en milieu urbain.

Les perspectives d'évolution de la demande en produits lactés, eu égard aux prévisions de développement économique du pays, tablent sur un rattrapage des recommandations internationales à

\section{Tableau I}

Evolution des prix du lait à la production et à la consommation au Maroc, et volumes importés

\begin{tabular}{|c|c|c|c|c|}
\hline Année & $\begin{array}{l}\text { Prix à la } \\
\text { production } \\
\text { (1) } \\
\text { (dirhams }\end{array}$ & $\begin{array}{c}\text { Prix à la } \\
\text { consommation } \\
(2) \\
\text { courants) }\end{array}$ & $\begin{array}{l}(1) /(2) \\
(\%)\end{array}$ & $\begin{array}{c}\text { Importations } \\
\text { (t équivalent lait) }\end{array}$ \\
\hline 1970 & 0,54 & 1,05 & 51,4 & 98230 \\
\hline 1975 & 0,90 & 1,20 & 75,4 & 102120 \\
\hline 1980 & 1,44 & 2,10 & 68,6 & 129960 \\
\hline 1985 & 2,05 & 3,10 & 66,1 & 128250 \\
\hline 1990 & 2,62 & 4,10 & 63,9 & 175440 \\
\hline 1995 & 2,94 & 5,00 & 58,8 & 306430 \\
\hline 2000 & 2,94 & 5,40 & 54,4 & 245260 \\
\hline 2005 & 2,94 & 6,20 & 47,4 & 252120 \\
\hline
\end{tabular}

D'après Sräri et coll., 2007 l'horizon 2020, ce qui reviendrait à doubler la production annuelle (14). Ceci a amené les pouvoirs publics à penser à l'intensification de l'élevage bovin dans les zones les plus propices : les périmètres irrigués et les régions pluviales favorables (plus de $400 \mathrm{~mm}$ de précipitations annuelles).

\section{Transformation industrielle du lait : d'une situation de rente à une compétition acharnée}

L'infrastructure de transformation du lait au Maroc s'est rapidement étoffée depuis les années 1970. La capacité de traitement installée est ainsi passée de 850000 1/jour en 1975 à 2700000 1/jour en 2004. Le tissu industriel s'est densifié avec une quarantaine d'unités de transformation réparties entre sociétés privées et coopératives (6). Quatre sous-filières peuvent être distinguées en fonction du type d'acteurs : la sous-filière industrielle organisée autour d'une usine privée de grande taille dont le principal acteur est la Centrale laitière ; la sous-filière coopérative avec l'émergence récente de la Copag, deuxième industrie laitière du Maroc ; la sous-filière semi-industrielle caractérisée par des opérateurs privés ou coopératifs de taille réduite ; et la sous-filière traditionnelle, basée sur la collecte informelle par colportage et des unités de transformation sommaire, comme les cafés et laiteries artisanales vendant directement leurs produits aux consommateurs.

Globalement, la situation de la transformation industrielle du lait au Maroc est très singulière par deux aspects qui la différencient de ses homologues dans les ensembles géographiques limitrophes : - les usines travaillent beaucoup plus avec du lait collecté à l'échelle du pays qu'avec de la poudre de lait importée, à la différence du cas voisin de l'Algérie (21) ; les importations moyennes annuelles de $14000 \mathrm{t}$ de poudre de lait entre 2002 et 2005 (13) représentent ainsi moins de 15 p. 100 des volumes de lait usinés ;

- l'existence de plusieurs unités industrielles de capacité importante (plus de 50000 t de lait traitées annuellement), induisant une organisation logistique et une gestion des flux et de la qualité du lait comme dans les pays développés, mais avec une différence de taille, à savoir un approvisionnement à partir d'une offre atomisée (des milliers d'élevages de taille réduite).

L'industrie laitière au Maroc apparaît aussi comme intermédiaire par rapport aux situations des laiteries artisanales en Afrique subsaharienne qui traitent des volumes limités et qui ont une activité

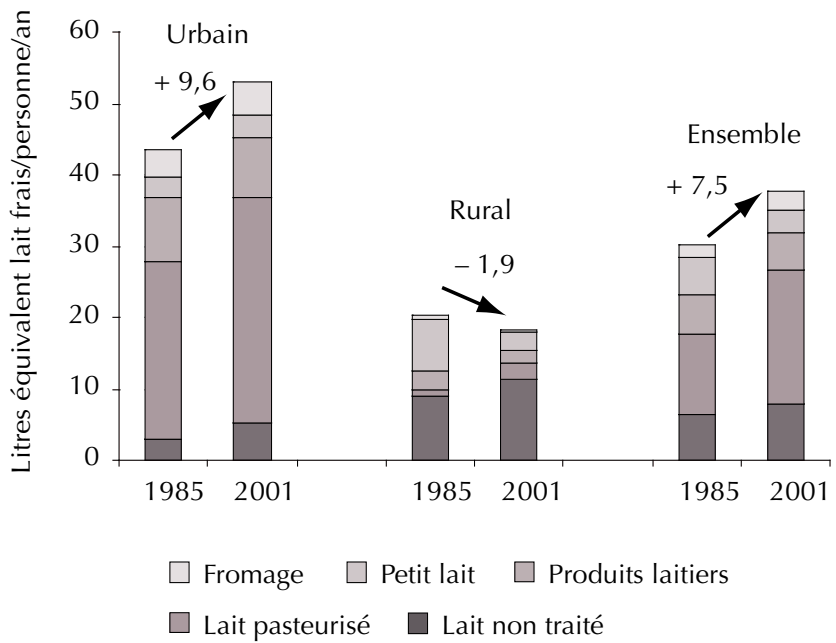

Figure 4 : évolution de la consommation de lait et produits laitiers au Maroc, par lieu de résidence. D'après le Haut Commissariat au Plan, Maroc, 2003. 
très saisonnière (5), ou bien celles des grandes usines qui s'approvisionnent auprès d'étables spécialisées, comme en Europe et en Amérique du Nord, avec des contrôles de qualité routiniers.

Actuellement, la filière industrielle est dominée par une unité, la Centrale laitière, qui se distingue des autres laiteries par sa taille. Elle collecte plus de 50 p. 100 du volume qui passe par les circuits formels de ramassage. Totalement privée, elle a noué d'importantes relations financières et techniques avec la multinationale Danone, devenue actionnaire dans son capital. Ce rapprochement, imposé par la recherche d'une compétitivité locale accrue, a induit l'émergence sur le marché marocain de produits laitiers dérivés qui bénéficient du label de qualité et des processus industriels associés au savoir-faire de la multinationale. Cependant, la montée en puissance d'une unité coopérative (Copag) a accru la concurrence sur le marché local des produits laitiers, depuis une dizaine d'années. Cette coopérative se distingue par son dynamisme récent et par un début d'intégration de la filière qui lui permettent de représenter aujourd'hui près de 20 p. 100 de la collecte du lait, part en croissance constante. De plus, son positionnement établi dans le créneau des dérivés laitiers de qualité (préparations laitières, yaourts, beurre...) distribués dans les supermarchés, sa situation géographique dans la zone du Souss, au sud ouest du Maroc, réputée pour l'esprit d'entreprise de sa population, et aussi le capital «sympathie » associé à son statut coopératif contribuent à consolider sa position.

Les unités industrielles opèrent généralement sur des bassins d'approvisionnement différenciés, mais parfois elles sont en compétition sur une même région. C'est le cas dans les zones jouxtant les grands centres urbains, notamment à la périphérie de la conurbation qui s'étale sur plus de 200 km, de Kenitra, Rabat Salé, Témara, Mohammedia, Casablanca à El Jadida, sur la façade côtière atlantique, groupant plus de 50 p. 100 des citadins du pays, et donc l'essentiel de la demande.

Les industriels ont aussi recours aux importations de poudre de lait, en particulier pour la production de produits laitiers à haute valeur ajoutée, afin de rattraper les déficits tant quantitatifs (en cas de mauvaise conjoncture climatique) que qualitatifs (correction des teneurs en matières grasses ou en protéines). Le déficit en matières grasses est aussi compensé par les importations de beurre qui avoisinent les $30000 \mathrm{t}$ en moyenne annuellement (sur la période 2002-2005) (13). Les volumes annuels importés sont cependant très variables (tableau I) selon les fluctuations de la production locale, elle-même fortement liée à la pluviométrie. Le renchérissement récent des cours mondiaux de la poudre de lait entier et écrémé, et du beurre risque d'accroître les coûts de fabrication des produits dérivés par les industriels, entraînant une réduction de leurs marges.

La majorité des autres structures coopératives de la sous-filière semi-industrielle souffre de parts de marchés réduites, avec une distribution uniquement régionale, et d'une gamme de produits peu attractive et non référencée dans les supermarchés. Certaines unités coopératives de transformation du lait éprouvent d'ailleurs de réelles difficultés financières, liées à une logistique de collecte peu rationnelle, ce qui les conduit souvent à ne pas rémunérer correctement leurs fournisseurs (les coopératives de collecte), plus particulièrement en périodes d'excédents laitiers (24). La hausse des prix de l'énergie fossile et du lait en poudre ainsi que l'obligation d'acquitter des impôts depuis l'année 2005 ne peuvent qu'accroître ces difficultés.

Les sous-filières industrielle et coopérative de grande taille essayent de développer des systèmes d'appui et de contrôle visant à améliorer la qualité du lait collecté. Les critères actuellement retenus par la législation (taux butyreux, densité et $\mathrm{pH}$ ) étant obsolètes et insuffisants, les industriels mettent en place d'autres tests plus onéreux (notamment le Delvotest ${ }^{\circledR}$ pour diagnostiquer la contamination par les antibiotiques), pour mieux évaluer la matière première qu'ils collectent. La sous-filière coopérative et dans une certaine mesure la sous-filière industrielle ont aussi comme objectif d'intégrer des fonctions d'appui technique aux producteurs (mise à disposition des coopératives d'éleveurs d'aliments composés adaptés aux divers types de fourrages utilisés, vulgarisation de l'ensilage de maïs et de pots trayeurs payables par les livraisons de lait, insémination artificielle, financement de sessions de formation...) visant à améliorer productivité et qualité. Les primes de qualité ou la redistribution des dividendes aux producteurs par la Copag vont aussi dans ce sens : fidéliser les éleveurs et sécuriser les approvisionnements.

\section{Collecte du lait : de l'essor volumétrique à l'inconnue qualitative}

Le plan laitier de 1975 comprenait de nombreuses mesures visant l'organisation de la collecte du lait par le lancement d'un vaste programme de construction et d'équipement de centres avec des bacs réfrigérants et leur mise à la disposition d'éleveurs organisés en coopératives (2). L'objectif était de permettre à des exploitations éparpillées à travers le pays et souvent éloignées de l'infrastructure de base (routes et réseau électrique) d'écouler quotidiennement le lait, produit périssable.

La multiplication des coopératives laitières pour la collecte du lait destiné aux usines a été un facteur majeur d'évolution tant quantitatif que qualitatif. Le nombre de centres de collecte de lait fonctionnels est passé de 10 en 1970 à 992 en 2006. Sur la même période, les volumes de lait drainés annuellement vers les usines ont évolué de 40 à plus de 800 millions de litres de lait. D'ailleurs, les augmentations du nombre de centres de collecte et des quantités de lait usiné affichent un remarquable parallélisme qui confirme que les efforts déployés dans le ramassage formel ont été l'élément majeur de la dynamique de livraison aux usines (figure 5). Le taux de lait usiné a ainsi fortement progressé de 10 à 60 p. 100 des volumes totaux, de 1970 à 2006, permettant à une très grande majorité d'exploitations, mêmes les plus modestes, d'intégrer la filière et de tirer des revenus quotidiens substantiels de l'élevage bovin. L'application des mesures d'ajustement structurel et le désengagement de l'Etat à partir des années 1980 n'ont donc pas eu d'incidence négative sur la dynamique de constitution des centres de collecte. Au contraire, étant donné le souci d'implication directe des élites locales dans la gestion de ces centres collectifs, véritables

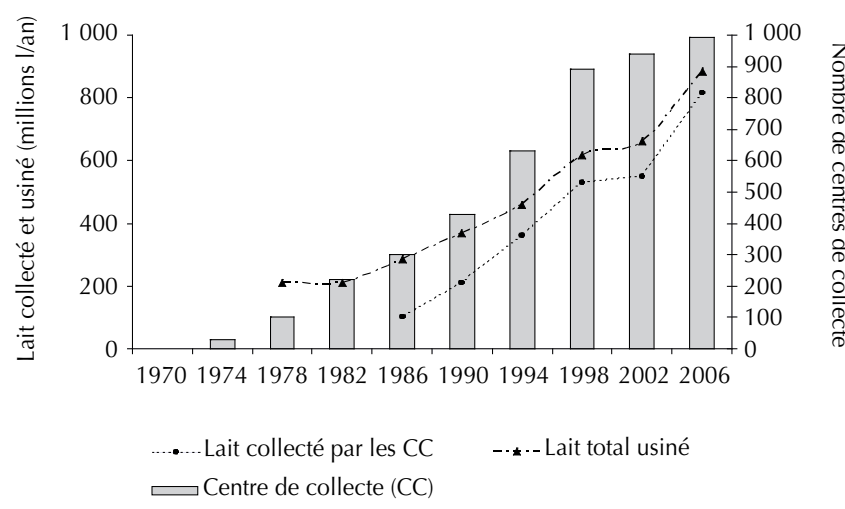

Figure 5 : évolution des effectifs des centres de collecte et des volumes de lait usinés. D'après le ministère de l'Agriculture, du Développement rural et des Pêches maritimes, 2006. 
tremplins vers d'autres fonctions de prestige, ils continuent de susciter un intérêt certain. Cet engouement se fait particulièrement ressentir lors de la tenue des assemblées générales annuelles de ces structures (26).

Aussi, le désengagement de l'Etat s'est-il beaucoup plus manifesté sur les modes de gestion technique des centres de collecte et sur leurs prestations globales que sur leurs effectifs. En effet, l'arrêt de l'implication des relais locaux de l'Etat a freiné l'ambition d'en faire des courroies de transmission de l'appui technique aux exploitations bovines qui y adhèrent. Par ailleurs, les changements issus de la libéralisation des prix du lait ont dissuadé certains éleveurs de continuer à écouler leur production dans les centres de collecte. En parallèle aux circuits formels de collecte du lait, des circuits plus courts ont émergé. La collecte du lait par colportage s'est développée intensément ces dernières années dans les bassins d'approvisionnement proches des grands centres urbains (12). Ceci est d'autant plus préoccupant que ces circuits ne bénéficient d'aucun contrôle sanitaire et sont même interdits par la législation. Mais la souplesse de paiement, l'absence d'évaluation de qualité et le passage des véhicules des colporteurs jusque dans les exploitations sont autant d'arguments qui plaident pour l'adoption de ce système de commercialisation par les éleveurs. Dans les bassins laitiers éloignés des grands centres urbains, les coopératives de collecte demeurent cependant les acteurs incontournables de l'écoulement du produit. Mais, le plus souvent, les membres de leurs bureaux dirigeants sont dépassés par l'importance des enjeux techniques (gestion des flux et de la qualité du lait, maîtrise des systèmes d'information relatifs à leurs activités quotidiennes) qu'ils doivent affronter (19), ce qui induit parfois de graves dysfonctionnements, voire des malversations financières.

Une des limites majeures de l'organisation actuelle de la collecte du lait réside dans son incapacité avérée à gérer les enjeux de qualité. L'atomisation de la production livrée jusqu'à deux fois par jour toute l'année et généralement mélangée dans des bacs réfrigérants collectifs, rend difficile le contrôle individualisé de la qualité. Par conséquent, les primes de qualité octroyées par les industriels aux coopératives ne peuvent pas être redistribuées aux adhérents selon la valeur de chaque lot livré. Elles sont juste restituées sous forme de dividendes au prorata du volume annuel livré, ou elles servent à financer des actions sociales (routes, dispensaires, écoles, frais de pèlerinage à la Mecque...) au profit de toute la communauté. Cette situation empêche toute action sérieuse sur les composantes de la qualité du lait, en l'absence d'un signal fort envers les exploitations d'élevage : pénalisation des fraudes (mouillage et écrémage) et des laits trop fortement contaminés en bactéries et/ou, à l'inverse, encouragement par des primes objectives des éleveurs qui consentent des efforts pour un lait ayant une bonne qualité hygiénique et riche en matières utiles. En l'absence de systèmes de distinction de la variabilité de la qualité du lait et de sa rémunération selon ses composants chimiques (taux butyreux et protéique) et hygiénique, la situation actuelle, comme le montrent les diagnostics réalisés à la ferme, est dominée par des livraisons de lots le plus souvent médiocres, surtout au plan bactériologique (22). Les échantillons analysés sont le plus souvent très chargés en bactéries $\left(>10^{6}\right.$ unités formant colonies $/ \mathrm{ml}$ ) en raison de pratiques d'hygiène rudimentaires (lavage sommaire, pas d'élimination des premiers jets, vaisselle laitière souillée...), de chaînes du froid embryonnaires de la ferme à l'usine, et de délais très longs entre la traite et l'entrée effective du lait dans la chaîne industrielle.

Avec ce mode d'organisation au sein de la filière, les coopératives de collecte assurent aussi, même théoriquement, une fonction d'articulation entre, d'une part, des éleveurs dispersés (plus de 700 000) et, d'autre part, les opérateurs de la transformation industrielle du lait, en aval. Mais il s'avère que, mise à part la fixation des prix du lait, imposés auparavant par les pouvoirs publics, il n'existe pas de véritable dialogue au sein de la filière. En effet, l'atomisation de la production empêche l'apparition d'espaces de concertation entre les industriels du lait et les éleveurs.

De même, aucune discussion sérieuse concernant la rémunération à la qualité n'est envisagée. Avec la libéralisation totale des prix du lait à la production et à la consommation en 1992, l'Etat n'assure plus qu'une autorité de contrôle, mais de facto, le prix à la ferme stagne, tandis que les industriels répercutent sur les consommateurs les augmentations successives des coûts des intrants qu'ils utilisent (énergie, emballages...), bénéficiant d'une situation de rente privilégiée.

\section{Elevage bovin laitier : de l'assistanat étatique au désengagement total de l'Administration}

L'essor de l'élevage bovin laitier, consécutif aux mesures productivistes retenues dans le cadre du plan laitier, a rapidement été freiné par l'entrée en vigueur, dès le début des années 1980, de politiques d'ajustement structurel imposées à l'économie marocaine. Ce changement de cap en matière de politique agricole a eu des effets marqués sur l'amont du secteur laitier : arrêt des subventions, excepté pour l'irrigation, chute du nombre d'étables soumises au contrôle laitier officiel (plus de 300 en 1990, moins d'une centaine en 2006) et cession progressive des prestations de santé animale et de l'insémination artificielle, qui deviennent payantes, au secteur vétérinaire privé.

Ces évolutions ont des conséquences sur les prix du lait à la production et à la consommation. A partir de 1980, la part de la valeur retirée par l'éleveur dans le prix final du lait chez le consommateur est en chute au profit de la marge des transformateurs du lait. Ces derniers récoltent en 2007 plus de 52 p. 100 de la valeur du lait à la consommation, pour moins de 30 p. 100 en 1980.

Le désengagement de l'Etat a aussi eu des répercussions drastiques sur l'organisation de la production de lait dans les exploitations agricoles. Tout d'abord, l'Etat a définitivement arrêté toute participation dans des sociétés para étatiques spécialisées dans l'élevage bovin (Comagri, ou Compagnie marocaine de gestion des exploitations agricoles) ou dans des sociétés agricoles qui disposaient d'un important cheptel (Sodea ou Société de développement agricole, avec un effectif total de près de 1500 vaches dans une dizaine d'étables). Certaines de ces unités, notamment celles de la Sodea, se caractérisaient par des performances zootechniques (plus de $6000 \mathrm{~kg}$ de lait/vache/an) et une rentabilité intéressantes, eu égard aux modes d'élevage adoptés : rareté des fourrages et recours massifs aux aliments concentrés (23). Toutefois, le diagnostic de la durabilité de ce genre d'ateliers révèle aussi des carences techniques manifestes : forts taux de mortalité, carrières écourtées des vaches, taux butyreux limités, importante contamination du lait par les antibiotiques...

Pour les exploitations agricoles privées, le désengagement de l'Etat a aussi induit des mutations dans les objectifs stratégiques d'élevage. Toutes les typologies fonctionnelles d'ateliers bovins dans différentes régions du Maroc, que ce soit dans les périmètres irrigués (plaine atlantique du Gharb et zone centrale du Tadla) ou les zones pluviales favorables (région périurbaine de Rabat - Salé, zone côtière atlantique de Ben Slimane...), montrent la rareté des exploitations spécialisées en production laitière, en dépit de la généralisation des vaches de type Holstein $(24,25)$. Dans les étables spécialisées, les vaches produisent plus de $4500 \mathrm{~kg}$ de lait annuellement, le plus souvent grâce à un recours massif aux aliments concentrés, leur permettant néanmoins de réaliser des 
marges financières annuelles satisfaisantes (aux alentours de 5000 dirhams/vache, soit près de 450 euros). Ces typologies révèlent aussi, dans tous les environnements de production (plaines irriguées et zones pluviales), la domination des exploitations bovines dites mixtes, généralement de petite taille (moins de cinq bovins sur une superficie inférieure à cinq hectares), où le croît des troupeaux (ventes de viande et augmentation des effectifs bovins par reproduction) constitue bien plus qu'un coproduit du lait. La différenciation de ces exploitations bovines repose ensuite sur l'efficience de l'usage des ressources alimentaires. Pour les plus performantes, en dépit de rendements laitiers modérés (moins de $3000 \mathrm{~kg}$ de lait/vache/an), la disponibilité de bases fourragères suffisantes conjuguée à une reproduction maîtrisée (1 veau/vache/ an) et à des croissances adéquates de la descendance permettent de dégager une marge brute annuelle positive par vache (jusqu'à 3000 dirhams, soit environ 270 euros).

En revanche, de très nombreuses exploitations bovines se caractérisent par des rendements laitiers par vache très faibles (parfois à peine $1000 \mathrm{~kg}$, même pour des femelles de type Holstein) en dépit d'un recours forcé à des aliments onéreux (les concentrés), luimême issu de la rareté des fourrages. Ces gaspillages des ressources alimentaires sont aussi la résultante de l'absence de l'usage des techniques de rationnement, puisque le désengagement de l'Etat a aussi eu pour conséquences d'éloigner définitivement les agents du développement de la majorité des exploitations bovines. Dans ces situations, la marge brute annuelle par vache est négative (jusqu'à 12000 dirhams de perte, soit 1050 euros). En moyenne, un suivi zootechnique rapproché de plus de 200 étables, représentatives des types d'élevage identifiés ci-dessus, dans diverses régions du Maroc, a montré que la marge brute par vache était de 1500 dirhams (environ 140 euros) en y incluant les ventes de lait et de bovins $(24,25)$.

Une autre caractéristique de tous les systèmes d'élevage bovin laitier au Maroc est leur sensibilité extrême à l'aléa climatique. Ce dernier se fait d'ailleurs ressentir même en zones irriguées. Jusqu'ici, les exploitations bovines laitières avaient comme stratégie de pallier les effets de la sécheresse en recourant en premier lieu aux stocks alimentaires issus des années favorables (la paille occupant à cet égard une place de choix), puis de se rabattre sur les aliments importés, comme l'orge, le maïs et les tourteaux de protéagineux (qui étaient peu onéreux) et, en cas de crise prolongée, de décapitaliser une part de leur cheptel (ventes d'animaux) pour sauvegarder l'outil de production. De même, les pouvoirs publics réagissaient par la mise sur le marché d'aliments importés dont les prix étaient largement inférieurs à ceux des produits locaux. L'envolée actuelle des cours des matières premières alimentaires sur le marché mondial, et un nouveau cycle de sécheresse prononcé lors de la campagne agricole 2006-2007 et à l'automne 2007 soulèvent désormais de nombreuses interrogations quant au maintien d'un élevage bovin paysan rentable ; les leviers classiques d'intervention étant devenus inopérants.

En effet, dans ce contexte, le prix de revient du kilogramme de lait a notablement augmenté et les éleveurs éprouvent des difficultés financières manifestes. Face au manque d'opportunités de négociation au sein de la filière, certaines coopératives d'éleveurs ont commencé à véhiculer l'idée de la cessation de la livraison du lait aux usines, espérant ainsi faire évoluer le rapport de forces et mettre la pression sur les unités industrielles pour qu'elles consentent à une augmentation du prix du lait.

Par ailleurs, le contexte de sécheresse aiguë récurrente, exacerbée par une compétition accrue pour l'usage des ressources hydriques entre différentes activités agricoles (élevage, arboriculture, marầchage...), voire extra-agricoles (tourisme et industrie), a aussi fait surgir la question de la valorisation de l'eau par l'élevage bovin laitier. Les premiers résultats (en moyenne $1,5 \mathrm{~m}^{3}$ d'eau par kilogramme de lait) montrent d'ailleurs que l'élevage laitier a encore des marges de progression importantes pour valoriser l'eau de manière optimale (27).

\section{PERSPECTIVES DES OPERATEURS} DE LA FILIERE LAITIERE EU EGARD

\section{A SA LIBERALISATION}

Les négociations des accords de libre-échange portent sur tous les produits agricoles, bruts ou transformés. Concernant la filière laitière marocaine, ces accords vont remettre en cause une des dernières protections dont elle bénéficie jusque-là : la taxation de plus de 100 p. 100 des importations de poudre de lait entier. En mettant sur un pied d'égalité les produits laitiers importés et ceux produits localement, les pouvoirs publics font le pari d'une adaptation de l'outil marocain de production et de sa mise à niveau. La libéralisation totale sera-t-elle l'option retenue et à quelle échéance ? Le Maroc pourra-t-il continuer à utiliser les droits de douane pour réguler l'entrée des produits importés ? Quelles incidences ces protections recouvrent-elles encore, à la lueur du renchérissement prononcé des cours mondiaux du lait en poudre et des matières premières alimentaires du bétail ?

Une analyse comparative des caractéristiques de l'élevage bovin au Maroc et en Europe montre qu'il existe des écarts fondamentaux entre les deux situations, aussi bien en termes de taille des structures impliquées, que des moyens mis en jeu pour la production (intrants, encadrement...), que de l'efficience technique de la production (tableau II). Ces différences, issues des efforts engagés en Europe depuis plus de 50 ans pour l'intensification de l'élevage bovin, trouvent aussi leurs explications dans un milieu climatique plus favorable, rendant les étables européennes bien moins vulnérables à la sécheresse que leurs homologues au Maroc, y compris celles des zones irriguées. De surcroît, même sans accident climatique, les éleveurs de bovins en Europe perçoivent des subventions significatives de près de 400 euros/vache/an (3).

\section{Tableau II}

Comparatif de la production laitière du Maroc et des pays de I'Union européenne à quinze

\begin{tabular}{|c|c|c|}
\hline Indicateur & Maroc & Union européenne \\
\hline $\mathrm{Nb}$. de vaches impliquées & $1600000 *$ & 21300000 ** \\
\hline Nb. d'exploitations laitières & 290000 & 736000 \\
\hline Nb. de vaches/exploitation & 5,5 & 29,0 \\
\hline Nb. d'ha/exploitation & $<7,5$ & 42,5 \\
\hline Nb. de vaches contrôlées & 6055 & 13560000 \\
\hline Lait/vache présente/an ${ }^{1}$ & 2000 à 4500 & $>6000$ \\
\hline $\begin{array}{l}\text { Charges alimentaires/ } \\
\text { charges totales (\%) }\end{array}$ & 70 à 75 & $<55 \%$ \\
\hline Nb. d'actifs/vache (UTH) ${ }^{2}$ & 0,24 & 0,06 \\
\hline \multicolumn{3}{|c|}{$\begin{array}{l}\text { * Femelles totales en production ; ** femelles dans des exploitations spécialisées } \\
\text { en lait } \\
{ }^{1} \text { Ne sont comptabilisées ici que les moyennes d'exploitations dans des systèmes à } \\
\text { finalité laitière } \\
2 \text { 'Exprimé en unités de travail humain, soit l'équivalent de } 300 \text { jours pleins de tra- } \\
\text { vail par an } \\
\text { D'après le ministère de l'Agriculture, du Développement rural et des Pêches mariti- } \\
\text { mes, 2006, et Chatellier et coll., } 2003\end{array}$} \\
\hline
\end{tabular}

mes, 2006, et Chatellier et coll., 2003 
L'intégration de la filière facilite en outre la transmission des signaux de prix et de qualité entre producteur et industriel. En Europe, les éleveurs peuvent livrer directement à l'industrie des quantités individuelles définies au préalable par les quotas, sans structure intermédiaire du type « centre de collecte».

De nombreuses industries du lait au Maroc traitant de faibles volumes de lait (moins de 10 millions de litres par an) ne peuvent réaliser les économies d'échelle permettant d'améliorer leur compétitivité. Ceci se fait particulièrement ressentir dans une conjoncture où l'innovation est limitée et où les produits à haute valeur ajoutée sont très peu présents dans la gamme de l'offre de l'industrie (6). En effet, près de 90 p. 100 du lait usiné est pasteurisé pour la boisson, ce qui génère des marges très ténues (tableau III). A contrario, en Europe, près de 65 p. 100 des volumes collectés sont transformés en fromages et yaourts, produits assurant les marges les plus élevées.

L'analyse de la filière laitière au Maroc et les éléments de comparaison avec l'Europe suggèrent qu'une série de chantiers très urgents guettent tous les opérateurs du secteur au Maroc. Ces réformes doivent être menées de front pour améliorer les performances globales et sécuriser l'approvisionnement des populations sans subir la compétition internationale. Trois axes prioritaires d'action se profilent par rapport aux faiblesses de la filière identifiées antérieurement.

\section{Centrer les efforts sur l'amélioration de la productivité et de la qualité}

Les niveaux actuels de qualité et de productivité par vache montrent de très importantes marges de progrès dans les élevages bovins laitiers, pour peu que des efforts d'appui technique soient adoptés. Bien entendu, cette option de l'appui technique à des milliers d'exploitations, détenant quelques bovins et disséminées à travers le territoire national, pose avec acuité la question des structures supposées mener à bien cette mission. A la lueur du désengagement de l'Etat, et donc de l'arrêt quasi définitif de l'implication de ses agents dans l'encadrement des producteurs, et suite à l'avènement d'associations d'élevage aux moyens humains et matériels souvent limités, l'appui technique généralisé pose un réel défi. Les modalités de cet appui technique, qui doit être de proximité (chaque exploitation ou groupe d'exploitations est un cas à part à étudier et à promouvoir), gagneraient à être précisées entre les opérateurs de la filière, avec l'assistance des autorités tutélaires (ministère de l'Agriculture et ses antennes régionales) et aussi en comptant sur l'intervention des organismes de recherche-développement. L'émergence d'organisations professionnelles performantes, prenant en charge l'appui technique (rationnement, suivis de la reproduction, conception de bilans fourragers prévisionnels, contrôle laitier...) et l'encadrement de leurs adhérents, apparaît comme un facteur indispensable à la promotion d'un élevage bovin laitier spécialisé rentable au Maroc, si toutefois elles disposent des éléments humains et des moyens logistiques nécessaires à leur mission. Le programme de mise à niveau des coopératives adhérentes de la Copag basé sur la création de complexes coopératifs à but économique, administratif et social ouvre de nouvelles perspectives dans ce sens.

Plus en aval, les efforts d'amélioration des performances et de la qualité supposent la mise à niveau des méthodes de collecte et de transformation du lait des industries industrielles peu performantes. La concentration de l'industrie laitière, par la fusion des unités de trop petite taille, apparaît comme inéluctable pour diminuer les charges de collecte. De même, la rationalisation des circuits logistiques est pressante. Dans les processus de transformation, la mise à niveau semble entamée par l'intervention des partenariats avec des multinationales qui ne peuvent qu'encourager les innovations sur le marché. D'autres pistes restent aussi ouvertes, comme la préservation de préparations laitières locales (les fromages blancs de type jben, le lben et le smen...).

\section{Gérer les ressources hydriques et leur valorisation par l'élevage bovin laitier}

Avec les niveaux actuels de disponibilité d'eau per capita (moins de $1000 \mathrm{~m}^{3}$ par an), la question de l'allocation des ressources

\section{Tableau III}

Performances comparées de la transformation laitière au Maroc et dans les pays de l'Union européenne à quinze

\begin{tabular}{|c|c|c|}
\hline Indicateur & Maroc & Union européenne \\
\hline Nb. d'unités industrielles & 48 & 5500 \\
\hline Laiteries de grande taille ${ }^{1}(\%)$ & $<10$ & 67 \\
\hline Lait collecté/lait produit (\%) & 50 & 93 \\
\hline Lait transformé en produits à boire (\%) & 87 & 21 \\
\hline Paiement à la qualité chimique & $\begin{array}{l}\text { Rudimentaire (uniquement le taux butyreux) } \\
\text { Gère difficilement le mouillage et l'écrémage } \\
\text { sur des livraisons individuelles } \\
\text { Paiement collectif qui ne favorise pas } \\
\text { la promotion de la qualité }\end{array}$ & $\begin{array}{l}\text { Contrôles routiniers et inopinés } \\
\text { Individualisé par exploitation } \\
\text { Taux butyreux, taux protéique, teneurs } \\
\text { en certains microconstituants } \\
\text { (caséines, minéraux...) }\end{array}$ \\
\hline Paiement à la qualité hygiénique & $\begin{array}{l}\text { Quasi inexistant } \\
\text { Ne favorise pas l'hygiène dans les étables } \\
\text { Impossibilité de détecter les étables qui } \\
\text { contamineraient des bacs collectifs avec } \\
\text { du lait renfermant des inhibiteurs de la flore }\end{array}$ & $\begin{array}{l}\text { Contrôles routiniers } \\
\text { Partie intégrante de la rémunération du lait } \\
\text { Contrôle systématique de la contamination } \\
\text { du lait par des inhibiteurs de la flore } \\
\text { (antibiotiques) }\end{array}$ \\
\hline
\end{tabular}

${ }^{1}$ Plus de $100000 \mathrm{t}$ de lait traitées annuellement

D'après la mission économique de l'ambassade de France à Casablanca, 2004, et El Ameli, 2005 
hydriques et de leur utilisation en agriculture (plus de 85 p. 100 des usages totaux) se pose avec une acuité croissante au Maroc et sera au cœur des débats futurs. L'élevage bovin en irrigué dispose d'atouts majeurs pour s'imposer (rôle social, trésorerie régulière...) mais il est en revanche très consommateur en eau, dans un contexte de tensions autour de l'usage de cette ressource. Si, à l'avenir, les pouvoirs publics étaient amenés à modifier les politiques en matière d'usage et de tarification de l'eau, l'élevage bovin serait en première ligne. Une augmentation du prix de l'eau risque en effet de réduire les marges au litre de lait. C'est un défi réel pour le secteur de l'élevage pour arriver à se maintenir par rapport aux spéculations qui le concurrencent. L'impact des politiques publiques futures en matière de gestion de l'eau mérite d'être analysé et pris en compte.

Les marges d'intervention techniques sont importantes : conversion des systèmes d'irrigation du gravitaire au goutte à goutte pour le maïs destiné à l'ensilage, maîtrise des itinéraires techniques des fourrages et augmentation de la productivité en lait par vache par les techniques du rationnement peuvent garantir des résultats probants. Mais cela suppose des investissements lourds et aussi de nouvelles formes de production que les éleveurs risquent de ne pas assimiler rapidement. Les impacts écologiques sont aussi à prendre en compte, dans des contextes souvent fragiles : aridité, érosion, salinisation des sols et des nappes phréatiques, par exemple. L'évolution technique devra aussi s'appuyer sur les nouvelles formes d'organisation professionnelle et d'appui aux producteurs. Enfin, une relocalisation de la production dans les zones plus favorables pourrait permettre de pallier les limites du développement laitier dans les régions très arides. Le récent positionnement de la Copag dans les plaines plus arrosées du Nord (Gharb), face à la pression sur la ressource en eau dans la région méridionale du Souss où la pluviométrie annuelle a été inférieure à $200 \mathrm{~mm}$ ces dix dernières années, va dans ce sens.

\section{Promouvoir une redistribution équitable des revenus tout le long de la filière}

La discussion des prix du lait au producteur et à la consommation demeure un véritable point de fixation des évolutions de la filière laitière. Face à l'envolée récente des prix des matières premières utilisées en élevage et à la stagnation des prix du lait au départ de l'exploitation, de nombreux éleveurs revendiquent une revalorisation significative de la rémunération du lait. D'ailleurs, au début de l'année 2008, la Centrale laitière, premier opérateur de collecte du lait, a consenti aux coopératives de collecte une augmentation de près de 0,35 dirhams ( 3 centimes d'euro environ, ce qui représente près de 12 p. 100 du prix antérieur) par kilogramme de lait dans certains de ses bassins de collecte, consciente de l'augmentation notoire des coûts de production et soucieuse de maintenir une activité d'élevage bovin pour garantir ses approvisionnements. Toutefois, ce même opérateur a répercuté au même moment sur les consommateurs l'intégralité des hausses des prix d'achat de la matière première.

A défaut, dans les zones où les industriels de la transformation n'ont pas encore augmenté le prix d'achat du lait auprès des coopératives de collecte, certains éleveurs ont déjà fortement reconverti en extensif leur mode de production. Il est donc impératif d'organiser au sein de la filière un débat serein sur la rémunération des efforts de chacun et aussi de paiement à la qualité des produits, pour sécuriser le maintien de l'activité d'élevage, et de garantir ainsi l'approvisionnement du marché, dans une conjoncture où l'importation de lait en poudre est devenue très onéreuse.

Ce débat ne peut faire l'économie de l'émergence d'une interprofession juste et représentative qui se charge de gouverner l'équité au sein de la filière laitière. Cependant, les marges de manœuvre sont faibles entre la nécessité de rémunérer le producteur et le faible pouvoir d'achat d'une majorité de consommateurs. En particulier, la filière pourra difficilement satisfaire la demande des consommateurs les plus démunis pour lesquels le prix actuel demeure trop élevé. Seules des mesures ciblées de soutien à ces populations - soutien au revenu par exemple - pourraient y remédier mais elles nécessitent d'harmoniser l'ensemble des politiques publiques liées aux aspects sociaux. En effet, les politiques de soutien à la consommation (sucre, céréales pour les produits alimentaires) se sont montrées peu efficaces pour cibler les plus démunis et leur coût budgétaire a explosé avec le renchérissement des cours mondiaux des matières premières.

L'amélioration de la rémunération des acteurs impose aussi d'augmenter la productivité et d'en répartir les gains. Des expériences d'intégration plus soutenue de la filière laissent entrevoir des gains de productivité qui pourraient bénéficier aux producteurs. Ainsi, dans le bassin laitier du Souss, la Copag est en phase d'expérimentation d'un atelier communautaire d'élevage des génisses de renouvellement pour ses adhérents. L'objectif est de s'affranchir de la dépendance vis-à-vis des importations de vaches, en dépit des risques sanitaires liés à cette concentration d'animaux. En effet, les cours ont aussi très fortement augmenté sur les marchés mondiaux, en particulier européens, en raison d'une demande croissante de pays comme la Russie. Toutefois, les impacts de ces expériences, toutes récentes, ne peuvent pas encore être objectivement évalués.

Au-delà des priorités de la filière laitière et des options générales leur correspondant, il apparaît aussi qu'une volonté politique globale de mise à niveau, par rapport aux standards internationaux de performances et de gouvernance, sera nécessaire. Les rôles de l'interprofession et des pouvoirs publics en termes de régulation et d'arbitrage au sein de la filière devront être spécifiés. Les pouvoirs publics devront être capables d'assister les différents opérateurs avec des moyens matériels et humains conséquents.

\section{CONCLUSION}

Dans l'optique de l'application d'un accord de libre-échange avec l'Union européenne, la filière laitière au Maroc se place en position de faiblesse. Elle est bien moins structurée que celles des pays européens, même si celles-ci montrent des limites en termes de durabilité d'élevage laitier : pollution des nappes et forte dépendance vis-à-vis des aliments importés notamment. De plus, quel que soit le maillon de la filière laitière marocaine considéré, son manque de performance est manifeste : des élevages peu spécialisés et avec de grandes marges d'amélioration de la productivité par vache, un système de collecte qui ne favorise pas la recherche de la qualité, une industrie peu innovante, dispersée, qui s'est surtout investie dans des transformations à marge limitée, et finalement une consommation en produits laitiers globalement insuffisante. Ces constats imposent à l'ensemble des opérateurs de la filière laitière marocaine de se pencher sur des options concertées de mise à niveau, tant au plan technique (plus de performances et plus de qualité en veillant à rationaliser les consommations en eau), qu'au plan organisationnel (la genèse d'une interprofession qui gère en harmonie les intérêts conjoints de tous les opérateurs), et même au plan humain (gros efforts de formation des intervenants). L'adoption de cette stratégie de mise à niveau doit se faire dans l'urgence pour continuer à faire face à une demande interne en croissance soutenue. En effet, outre la croissance démographique qui ne fléchit pas, la volonté d'ouverture touristique du pays, avec dix millions de visiteurs attendus à l'horizon 2010, augmenterait significativement la demande en produits lactés. Par ailleurs, avec les 
choix politiques du pays qui convergent vers le libre-échange, il y a lieu de s'interroger sur la nature des responsabilités de chacun des acteurs de la filière dans un contexte de désengagement de l'Etat. Outre l'adoption de bonnes pratiques à tous les échelons de la filière laitière, de l'élevage bovin à la transformation industrielle, un effort doit aussi être consenti par l'ensemble pour l'émergence d'opportunités de discussion et de négociation autour des enjeux vitaux : prix, qualité, saisonnalité... A cet égard, les négociations en cours de l'accord de libre-échange avec l'UE constituent aussi une occasion propice pour l'adoption au sein de la filière laitière marocaine de ce nouveau cadre de concertation. L'Etat et ses services, ainsi que les opérateurs industriels jouant un rôle moteur d'intégration de la filière et de représentation des producteurs coopératives - sont appelés à y jouer un rôle déterminant.

\section{Remerciements}

Un grand merci aux divers intervenants de la filière laitière au Maroc (associations d'éleveurs, transformateurs du lait, pouvoirs publics...) d'avoir accepté de discuter des perspectives d'évolution.

\section{BIBLIOGRAPHIE}

1. BENJELLOUN S., ROGERS B.L., BERRADA M., 1998. Income and consumption effects of milk commercialization in the Lukkos area of Morocco. Ecol. Food Nutr., 37: 269-296.

2. BOURBOUZE A., 2002. Les temps longs du développement : patientes transformations des systèmes d'élevage au Maghreb. In : Dufumier M. éd., Un agronome dans son siècle. Actualité de René Dumont. Paris, France, Karthala, p. 95-107.

3. CHATELLieR V., COLSON F., FUENTES M., VARD T., 2003. Les exploitations d'élevage herbivore dans I'Union européenne. Prod. Anim., $13: 201-213$

4. COLEOU J., 2005. L'approvisionnement en produits animaux et ses enjeux géostratégiques. In : Théwis A., Bourbouze A., Compère R., Duplan J.-M., Hardouin J., coords., Manuel de zootechnie comparée Nord-Sud. Paris, France, AUF/Inra, p. 89-114.

5. CORNIAUX C., DUTEURTRE G., DIEYE P.N., POCCARD-CHAPUIS R., 2005. Les minilaiteries comme modèle d'organisation des filières laitières en Afrique de l'Ouest : succès et limites. Revue Elev. Méd. vét. Pays trop., $\mathbf{5 8}: 237-243$.

6. EL AMELI L., 2005. Les industries alimentaires au Maroc : dynamiques et perspectives de développement. Rabat, Maroc, ministère de l'Education nationale, de l'Enseignement supérieur, de la Formation des Cadres et de la Recherche scientifique, $464 \mathrm{p}$.

7. EL KHYARI T., 1985. Agriculture au Maroc. Casablanca, Maroc, Okad, $500 \mathrm{p}$.

8. FABRE P., 1997. Manuel d'analyse financière et économique des projets de développement. Bruxelles, Belgique, Commission européenne, 380 p. (Série Méthodes et instruments pour la gestion du cycle de projet)

9. FAO, 1998. Le lait et les produits laitiers dans la nutrition humaine Rome, Italie, FAO, 223 p. (Coll. Alimentation et nutrition, $\mathrm{n}^{\circ} 28$ )

10. FAYE B., ALARY V., 2001. Les enjeux des productions animales dans les pays du Sud. Prod. Anim., $14: 3-13$.

11. HAUT COMMISSARIAT AU PLAN, 2003. Enquête nationale de consommation et de dépenses des ménages 2000-2001. Rabat, Maroc, Haut Commissariat au Plan, 189 p.

12. LETHEUIL H., 1999. La filière lait à Casablanca : approvisionnement, typologie des unités de transformation, commercialisation. Mémoire $3^{\mathrm{e}}$ cycle. Montpellier, France, Cnearc, $156 \mathrm{p}$.

13. MADRPM, 2006. Situation de I'agriculture marocaine, vol. 1. Rabat, Maroc, ministère de l'Agriculture, du Développement rural et des Pêches maritimes, $127 \mathrm{p}$.

14. MADRPM, 2000. Stratégie de développement de l'élevage, tome II, Filières de production animale. Rabat, Maroc, ministère de l'Agriculture, du Développement rural et des Pêches maritimes, $68 \mathrm{p}$.

15. MARA, 1975. Le plan laitier. Rabat, Maroc, ministère de l'Agriculture et de la Réforme agraire, $115 \mathrm{p}$.

16. MISSION ECONOMIQUE DE L'AMBASSADE DE FRANCE A CASABLANCA, 2004. Fiche de synthèse. Le marché des produits laitiers au Maroc. Www.missioneco.org/maroc/documents_news.as?/V=7_ PDF_75845 (consulté 10.03.2005).

17. MOUSTIER P., LEPLAIDEUR A., 1999. Méthodes d'analyse des filières vivrières en Afrique. Montpellier, France, Cirad, 36 p. (Coll. Alimenter les villes)

18. OCDE/FAO, 2006. Perspectives agricoles 2006-2015. http://www. oecd.org/dataoecd/32/50/35018755.pdf (consulté 03.04.2007)

19. OUDIN E., 2006. Analyse et propositions d'améliorations du fonctionnement de coopératives de collecte laitière au Maroc : approche par les systèmes d'information. Mémoire fin d'études, INA Paris-Grignon, France, $115 \mathrm{p}$.

20. PROJET SEBOU, 1961. Mise en valeur agricole de la plaine du Gharb, fascicule 4, livret 2. Rabat, Maroc, ministère de l'Agriculture et de la Réforme agraire, $85 \mathrm{p}$.

21. SRAIRI M.T., ELLOUMI M., BEN SALEM M., MADANI T., YAKHLEF H., BOURBOUZE A., FAYE B., 2007. Analyse comparée de la dynamique de la production laitière dans les pays du Maghreb. Cah. Agric., 16 : 251-257.

22. SRAIRI M.T., HASNI ALAOUI I., HAMAMA A., FAYE B., 2005. Relations entre pratiques d'élevage et qualité globale du lait de vache en étables suburbaines au Maroc. Revue Méd. vét., 156 : 155-162.

23. SRAIRI M.T., KESSAB B., 1998. Performances et modalités de production laitière dans six étables spécialisées au Maroc. Prod. Anim., 11 : 299-304.

24. SRAIRI M.T., LEBLOND J.M., BOURBOUZE A., 2003. Production de lait et/ou de viande : diversité des stratégies des éleveurs de bovins dans le périmètre irrigué du Gharb au Maroc. Revue Elev. Méd. vét. Pays trop., 56: $177-186$.

25. SRAIRI M.T., LYOUBI R., 2003. Typology of dairy farming systems in Rabat suburban region, Morocco. Arch. Zootech., 52: 47-58.

26. SRAIRI M.T., MEDKOURI H., 1999. Production et écoulement du lait en région d'agriculture pluviale au Maroc. Tropicultura, 16-17 : 321-326.

27. SRAIRI M.T., TOUZANI I., KUPER M., LE GAL P.-Y., 2007. Valorisation de l'eau d'irrigation par la production bovine laitière dans un périmètre de grande hydraulique au Maroc. Cah. Agric., 17 : 271-279.

28. TAHIRI M., 1963. Structures agraires et industries agricoles. In : Dresch J., Attek M., Tiano A., Tahiri M., de Bernis G., Khodja K.A., Siksou M., Lahbabi M., Borella F., Belal A., Serfaty A., Bouabid A., éds, Industrialisation au Maghreb. Paris, France, François Maspero, p. 75-92.

29. TALHA L., 1994. Croissance, crise et mutations économiques au Maghreb. In : Bichara K., éd., Alternatives Sud : ajustement structurel au Maghreb. Belgique, Louvain La Neuve, John Libbey, p. 27-80.

Reçu le 19.04.2007, accepté le 08.04.2008 


\section{Summary}

Sraïri M.T., Chohin Kuper A. Consequences of Market Liberalization for the Operators of the Dairy Subsector in Morocco

The Moroccan dairy subsector has gone through an eventful recent history. The initial situation, in the early 1970s, was characterized by a high level of State regulation. At that time, there was an increasing domestic demand associated to a low availability of milk products; therefore State authorities encouraged local production and processing. But at the beginning of the 1980s, a series of structural adjustment measures were implemented and all subsidies were progressively suppressed. These sudden successive changes seriously impacted on the organization of the dairy subsector, particularly at the level of dairy cattle farms, by markedly modifying production practices. Currently, another significant stage for the Moroccan dairy subsector is the ongoing negotiation of free trade agreement with the European Union. This will lead to the end of protection for domestic dairy products and to increased competition between local and imported dairy products. In a context of market liberalization associated with the price of agricultural inputs rising on international markets, it will be essential to upgrade productive tools and policies of the subsector, which will have consequences on all operators along the commodity chain. This will be vital for the upholding of milk production, collection and transformation activities in Morocco, under conditions favorable to their sustainable development: pursuit of optimal milk yield and quality, efficient irrigation water productivity, and fair distribution of incomes generated by the subsector to all operators, with an aim to adjust the price to the purchase power of consumers.

Keywords: Cow milk - Trade liberalization - Quality - Animal husbandry - Morocco.

\section{Resumen}

Sraïri M.T., Chohin Kuper A. Consecuencias de la liberalización de mercados para los operadores de la filial lechera en Marruecos

La filial lechera marroquí ha conocido una evolución agitada desde el principio de los años 1970 hasta nuestros días. La situación inicial, muy protegida, se caracterizaba por un marcado intervencionismo del estado, debido a la necesidad de paliar urgentemente la escasez de leche frente a una demanda en pleno auge. A partir de 1980, la aplicación de medidas de ajuste estructural suprimió progresivamente toda forma de subvención. Estos trastornos y la rapidez con la cual se dieron, tuvieron repercusiones marcadas sobre la organización de la filial, particularmente en los establecimientos de bovinos lecheros, en donde las características de producción han evolucionado notablemente. Actualmente, se avanza un paso más con la preparación a la entrada en vigor de los acuerdos de libre comercio con la Unión Europea. Esto provocará el fin de toda protección y de un aumento de la competencia entre productos lecheros locales e importados en el mercado marroquí. En esta óptica de liberalización y teniendo en cuenta la inflación actual de los precios de los insumos agrícolas en los mercados internacionales, se espera una nivelación necesaria de los útiles de producción y de regulación en el seno de la filial, con consecuencias ciertas para el conjunto de los operadores. Está en juego el mantenimiento de las actividades de producción, de colecta y de transformación de leche en Marruecos bajo condiciones propicias a su desarrollo duradero: búsqueda de la productividad óptima y de productos de calidad, eficiencia de la valorización del agua, distribución equilibrada de los ingresos de la filial a todos sus participantes, con el objetivo de un precio al consumidor en acuerdo con el poder de adquisición.

Palabras clave: Leche de vaca - Calidad - Liberalización de intercambio - Ganadería - Marruecos. 